\title{
Multiple attempts to stop smoking, even with relapses, reduced loss of pulmonary function
}

\author{
Murray RP, Anthonisen NR, Connett JE, et al for the Lung Health Study Research Group. Effects of multiple attempts to quit smoking \\ and relapses to smoking on pulmonary function. J Clin Epidemiol 1998 Dec;51:1317-26.
}

\section{Question}

What are the effects of multiple failed attempts to stop smoking on pulmonary function of adults?

\section{Design}

Retrospective analysis of a cohort of participants involved in a randomised controlled trial (Lung Health Study), with annual follow up for 5 years.

\section{Setting}

10 clinical centres in North America**

\section{Participants}

5887 adults aged 35-60 years, who smoked and had evidence of early chronic obstructive pulmonary disease (ie, had an $\mathrm{FEV}_{1} / \mathrm{FVC}$ [forced expiratory volume at $1 \mathrm{sec} /$ forced vital capacity] interval of $\leqslant 70 \%$, and an $\mathrm{FEV}_{1}$ of $55-90 \%$ of predicted normal).

\section{Assessment of risk factors}

Smoking status was assessed at baseline and at 5 annual visits thereafter. Participant reports of abstinence were confirmed by salivary cotinine concentrations $(<20 \mathrm{ng} / \mathrm{ml})$ or if nicotine substitution therapy was used, by CO concentrations $(<10 \mathrm{ppm}$ in end tidal expired air). Participants were classified as sustained quitters (verified non-smokers at the first follow up visit, who remained abstinent at all subsequent follow up visits, and who recalled no month with average smoking $>1$ cigarette/d, starting 4 mo after study entry); continuous smokers (reported smoking at all 5 annual visits), and intermittent smokers (verified non-smokers at $\geqslant 1$ annual visit and reported smoking at $\geqslant 1$ annual visit).

\section{Main outcome measures}

$\mathrm{FEV}_{1}$ assessed using spirometry (after bronchodilator use) and change in $\mathrm{FEV}_{1}$ reported as a percent of predicted normal, adjusted for African-American and Asian participants.

\section{Main results}

149 participants who died, 66 participants who reported lung cancer during follow up, and 21 participants with extreme $\mathrm{FEV}_{1}$ values were excluded from the analysis. At 5 years, sustained quitters had the highest $\mathrm{FEV}_{1}$ (79.8), followed by intermittent quitters (77.9), and continuous smokers (73.1). From years 1 to 5, sustained quitters had the smallest annual rate of loss in $\mathrm{FEV}_{1}$ predicted (loss of $0.33 \% / y$ ), followed by intermittent quitters (loss of $0.58 \% / y$ ), and continuous smokers (loss of $1.18 \% / y$ ). Continuous smokers lost the most lung function at all doses of cigarettes; intermittent smokers who had 1 change in smoking status did better than continuous smokers, and those who had $\geqslant 2$ changes did even better. Multivariate analysis found that men who were sustained and intermittent quitters had better $\mathrm{FEV}_{1} \%$ predicted but no difference in $\mathrm{FEV}_{1}$ change when compared with continuous smokers. Women had the same pattern for $\mathrm{FEV}_{1} \%$ predicted but intermittent smokers had a lower $\mathrm{FEV}_{1}$ than continuous smokers.

\section{Conclusion}

Adults who attempted to stop smoking (and may have relapsed) lost less pulmonary function, and lost function less rapidly, than people who smoked continuously, given the same average dose of cigarettes over a 5 year period.

*Anthomisen NR, Connett JE, Kiley JP, et al for the Lung Health Study Research Group. JAMA 1994;272:1497-505.

Sources of funding:Division of Lung Diseases of the National Heart, Lung, and Blood Institute; Boehringer Ingelheim Pharmaceutical, Marion Merrell Dow, and Merrell Dow Pharmaceuticals (Canada) contributed drugs used by the study.

For correspondence: Dr R P Murray, Section of Clinical Epidemiology and Outcome Evaluation, Room MS754, Health Sciences Centre, University of Manitoba, 820 Sherbrook Street, Winnipeg, Manitoba R3A 1R9, Canada. Fax +1 2047893905.

\section{Commentary}

Despite broad consensus that stopping smoking reduces the decline in lung function caused by smoking, little has been published on the effects of relapsed smoking cessation. One study found that participants who attempted to stop smoking and then relapsed had steeper rates of decline in $\mathrm{FEV}_{1}$ compared with participants who continued to smoke without attempting to stop, ${ }^{1}$ which contradicts the findings of this study by Murray et al.

The authors used data from a randomised controlled trial of a smoking cessation intervention to examine the association between multiple smoking cessation attempts with relapses to smoking and pulmonary function in adults. The study design was robust-inclusion and exclusion criteria were explicit, as were those for the construction of the study categories (ie, quitters, continuous smokers, and intermittent smokers). The authors accounted for sex differences, ethnicity, number of cigarettes smoked, and for potential errors in lung function measurement by using spirometry. They excluded patients who died during the study or were diagnosed with lung cancer. Smoking cessation was verified using either salivary cotinine levels or CO concentrations, thus eliminating the potential for participant misreporting of smoking.

The results of the study can be used by nurses in the context of providing individual advice and public health education on the benefits of smoking cessation. Smokers who attempted to stop smoking but relapsed lost less pulmonary function and lost pulmonary function less rapidly than people who did not attempt to stop: in other words, trying to stop smoking was beneficial, even if it was not maintained. Also, Murray et al suggest that women seemed to benefit more from stopping smoking than men because, for some reason, women's lung function deteriorates more rapidly than men's with continued smoking.

Graham R Williamson, RGN, MA Senior Lecturer Institute of Health Studies University of Plymouth Exeter, Devon, UK

1 Sherrill DL, Enright P, Cline M, et al. Chest 1996;109:1001-5. 